\title{
Grow-up of critical solutions for a non-local porous medium problem with Ohmic heating source
}

\author{
Evangelos A. Latos and Dimitrios E. Tzanetis
}

\begin{abstract}
We investigate the behaviour of solution $u=u(x, t ; \lambda)$ at $\lambda=\lambda^{*}$ for the non-local porous medium equation $u_{t}=\left(u^{n}\right)_{x x}+\lambda f(u) /$ $\left(\int_{-1}^{1} f(u) \mathrm{d} x\right)^{2}$ with Dirichlet boundary conditions and positive initial data. The function $f$ satisfies: $f(s),-f^{\prime}(s)>0$ for $s \geq 0$ and $s^{n-1} f(s)$ is integrable at infinity. Due to the conditions on $f$, there exists a critical value of parameter $\lambda$, say $\lambda^{*}$, such that for $\lambda>\lambda^{*}$ the solution $u=$ $u(x, t ; \lambda)$ blows up globally in finite time, while for $\lambda \geq \lambda^{*}$ the corresponding steady-state problem does not have any solution. For $0<\lambda<\lambda^{*}$ there exists a unique steady-state solution $w=w(x ; \lambda)$ while $u=u(x, t ; \lambda)$ is global in time and converges to $w$ as $t \rightarrow \infty$. Here we show the global grow-up of critical solution $u^{*}=u\left(x, t ; \lambda^{*}\right)\left(u^{*}(x, t) \rightarrow \infty\right.$, as $t \rightarrow \infty$ for all $x \in(-1,1))$.
\end{abstract}

Mathematics Subject Classification (2000). Primary 35K55; Secondary 35B05.

Keywords. Non-local parabolic problems, Porous medium, Grow-up of solutions.

\section{Introduction}

In the present work we prove the global "grow-up" of critical solutions for the following non-local problem:

$$
\begin{gathered}
u_{t}-\left(u^{n}\right)_{x x}=\frac{\lambda f(u)}{\left(\int_{-1}^{1} f(u) \mathrm{d} x\right)^{2}}, \quad x \in \Omega=(-1,1), \quad t>0 \\
u(1, t)=0, \quad u(-1, t)=0, \quad t>0 \\
u(x, 0)=u_{0}(x)>0, \quad x \in \Omega .
\end{gathered}
$$

E. A. Latos was supported by the Greek State Scholarship Foundation (I.K.Y.). 
Here $\lambda>0$ and $u$ is a (classical) solution $\left(u \in C^{2}\left(\Omega_{T}\right) \cap C\left(\bar{\Omega}_{T}\right)\right.$, for some $T>0)$ to $(1)$, provided that $u_{0}(x) \in C(\bar{\Omega})$ where $\Omega_{T}=(-1,1) \times(0, T)$.

On the other hand, the function $u:[0, T] \rightarrow L^{1}(\Omega)$, is called a weak or $L^{1}$-solution to problem (1) if $u \in C\left([0, T] ; L^{1}(\Omega) \cap L^{\infty}\left(\Omega_{T}\right)\right)$ for some $T>0$ and satisfies:

$$
P(u ; \eta) \equiv \int_{\Omega_{T}}\left[u \eta+u^{n} \eta_{x x}+\lambda \frac{\eta f(u)}{\left(\int_{\Omega} f(u) \mathrm{d} x\right)^{2}}\right] \mathrm{d} x \mathrm{~d} t+\int_{\Omega} u_{0}(x) \eta(x, 0) \mathrm{d} x=0,
$$

where $\eta=\eta(x, t) \in C_{0}^{\infty}\left(\bar{\Omega}_{T}\right)$.

The corresponding steady-state problem to (1) is:

$$
\begin{aligned}
& \left(w^{n}\right)^{\prime \prime}+\mu f(w)=0, \quad x \in(-1,1), \\
& w(1)=w(-1)=0,
\end{aligned}
$$

where $w=w(x)=w(x ; \mu)$ and $\mu=\lambda /\left(\int_{-1}^{1} f(w) \mathrm{d} x\right)^{2}$ is referred as the local parameter, while $\lambda$ as the non-local one.

Setting $z=w^{n}, w$ is called a classical solution (in the sense $w \in C^{2}(\Omega) \cap$ $C(\bar{\Omega}))$ to $(2)$ if $z$ is a classical solution to

$$
\begin{aligned}
& z_{x x}+\mu \phi(z)=0, \quad x \in(-1,1), \\
& z(1)=z(-1)=0
\end{aligned}
$$

where $\phi(z)=f\left(z^{1 / n}\right)$.

The nonlinear function $f(s)=\phi\left(s^{n}\right)$ satisfies:

$$
\begin{aligned}
& f(s)>0, \quad f^{\prime}(s)<0, \quad s \geq 0, \\
& \int_{0}^{\infty} s^{n-1} f(s) \mathrm{d} s<\infty,
\end{aligned}
$$

$n \geq 1$. The parameter $\lambda$ is positive and there exists a critical value (due to $4 \mathrm{~b},[15])$, say $\lambda^{*}$, such that to each $\lambda>\lambda^{*}$ the solution $u=u(x, t ; \lambda)$ blows-up in finite time and there is not any steady-state solution $w$ for $\lambda \geq \lambda^{*}$, while to each $\lambda<\lambda^{*}$ there exists a unique steady-state solution $w$ and the solution $u$ is global in time. The complete response $\mu$-diagram (local) and $\lambda$-diagram (non-local) to problem (3) can be obtained by quadratures, see [15, p.448].

By "global grow-up" of critical solutions $u^{*}$ we mean that the solution $u^{*}=u\left(x, t ; \lambda^{*}\right)$ to problem (1) is global in time and diverges at infinity globally in space, in the sense that $u^{*}(x, t) \rightarrow \infty$ as $t \rightarrow \infty$ for all $x \in(-1,1)$.

Condition (4a) permits us to use direct comparison methods, while (4b) implies bounded $\lambda$-spectrum $\left(0<\lambda<\lambda^{*}<\infty\right)$ for the corresponding nonlocal steady-state problem, see $[8,9,13,14]$. On the other hand, if $f^{\prime}(s)>0$ then a direct comparison does not hold, so for comparison purposes we have to use lower-upper solution pair, see [10]. We denote the solution to (1) by $u=u(x, t)=u(x, t ; \lambda)$. For simplicity we assume $u_{0}(x)$ to be continuous, $u_{0}(1)=u_{0}(-1)=0$ and $u_{0}(x)>0$ for $x \in(-1,1)$, the latter requirement is needed in order to take strictly positive solutions and to avoid degeneracy of problem (1).

Our motivation to address (1), concerning the conduction term $\Delta u^{n}$ (or $\nabla \cdot u^{n-1} \nabla u$ ) comes from [15]. In [15], the "plasma heating" equation 
$u_{t}=\left(u^{3} u_{x}\right)_{x}+\lambda f(u) /\left(\int_{-1}^{1} f(u) \mathrm{d} x\right)^{2}$ is discussed; more precisely the conduction term $\left(u^{4}\right)_{x x}$ or $\left(u^{3} u_{x}\right)_{x}$ is introduced, where the term $u^{3}$ accounts for heat transport dominated by thermal radiation by assuming the Stefan-Boltzman law for emission of thermal radiation. Actually, in this view Eq. (1) is a generalization of the plasma heating equation.

On the other hand, if $u$ represents the fluid density of a compressible fluid through a porous medium, then Darcy's law can lead to the equation:

$$
u_{t}=\nabla \cdot \sigma(u) \nabla u, \quad \sigma(u)=K^{\prime}(u),
$$

which is the filtration equation. For the latter equation specializing to an isothermal perfect gas, we can obtain the porous medium equation:

$$
u_{t}=\nabla \cdot\left(u^{n} \nabla u\right), \quad n>0, \quad \text { or } u_{t}=\Delta u^{n}, \quad n>1 .
$$

The Eq. (6) also models the thickness $u$ of a viscous drop spreading under gravity over horizontal surface as well as the horizontal spreading of highly fissured volcanos, see for details [12, p.216].

Concerning the non-local reaction term of problem (1), this comes from modelling Ohmic heating phenomena as well as in shear bands of metals which are being deformed at high strain rates (see [2-4]), in the theory of gravitational equilibrium of polytropic stars [7], in the investigation of the fully turbulent behaviour of flows, using invariant measures for the Euler equation [5].

In the plasma heating problem $\left(\left(u^{n}\right)_{x x}\right.$ for $n=4$, see [15]), for $0<\lambda<\lambda^{*}$ there exists a unique global in time solution $u=u(x, t ; \lambda) \rightarrow w(x ; \lambda)$, for any $x \in(-1,1)$ as $t \rightarrow \infty$, where $w(x ; \lambda)$ is the corresponding unique steady-state solution to problem (1). While, for $\lambda \geq \lambda^{*}$ there is no any solution $w=w(x ; \lambda)$, actually $w(x ; \lambda) \rightarrow \infty$ as $\lambda \rightarrow \lambda^{*}-$ for all $x \in(-1,1)$, see the corresponding response (bifurcation) diagram in [15]. The solution $u=u(x, t ; \lambda)$ blows up globally in finite time for $\lambda>\lambda^{*}$, i.e. $u(x, t) \rightarrow \infty$ for all $x \in(-1,1)$ and $u_{x}( \pm 1, t) \rightarrow \mp \infty$, as $t \rightarrow t^{*}-<\infty$. All the results in [15], given for $n=4$, can be generalized straightforward by the same way for any $n>1, n \in \mathbb{N}$.

Our main purpose in this article is to prove the global "grow-up" of solution $u^{*}=u\left(x, t ; \lambda^{*}, u_{0}^{*}\right)$ to problem (1). Therefore, we make a number of comparisons, namely we get: $u^{* n}(x, t)<U^{n}\left(x, t, \lambda^{*}, u_{0}\right)=z(x, t)<\bar{u}(x, t)=$ $\widehat{u}(x, \tau)$, where $u^{*}, z$ are lower solutions to $U, \bar{u}$-problems, respectively, while $\widehat{u}(x, \tau)$, with rescaling time $t=t(\tau)$, satisfies the non-local heat equation for which we know that it is a global in time solution. This result is given in the following section.

\section{Grow-up of critical solutions $\left(\lambda=\lambda^{*}\right)$}

Firstly we define (classical) upper and lower solutions to problem (1). An upper (lower) solution to problem (1) is any function $\bar{u}(\underline{u})$ which satisfies (1) by substituting $\geqslant(\leqq)$ for $=$.

We also need to impose an extra condition on $f$, (see [6]). Thus we define:

$$
f(s)=f\left(\sigma^{1 / n}\right)=\phi(\sigma), \quad s=\sigma^{1 / n} \text { and } \Psi(\sigma)=\frac{\phi(\sigma) \sqrt{\mu(\sigma)}}{\Phi(\sigma)},
$$


where $\int_{0}^{\infty} s^{n-1} f(s) \mathrm{d} s=\frac{1}{n} \int_{0}^{\infty} \phi(\sigma) \mathrm{d} \sigma<\infty \quad($ due to $(4 \mathrm{~b}))$ and

$$
\Phi(\sigma)=\int_{\sigma}^{\infty} \phi(p) \mathrm{d} p, \quad \mu(\sigma)=\frac{1}{2}\left(\int_{0}^{\sigma} \frac{\mathrm{d} p}{\sqrt{\Phi(p)-\Phi(\sigma)}}\right)^{2} .
$$

Finally the function $\phi$ satisfies:

$$
\begin{aligned}
& \liminf _{\sigma \rightarrow \infty} \Psi(\sigma)>C>0, \text { and } \lim _{\sigma \rightarrow \infty} \mu(\sigma) \phi(\sigma)=C_{0}>0, \\
& \text { or } \lim _{\sigma \rightarrow \infty} \frac{\mu(\sigma) \phi(\sigma)}{\sigma}=C_{1}>0 .
\end{aligned}
$$

For instance, a function satisfying (7a) is: $\phi(\sigma)=e^{-\sigma}$, while for (7b) is $\phi(\sigma)=$ $b(1+\sigma)^{-1-b}, C_{1} \leq 2, b>0$; for these type of conditions, see also $[6,11]$.

Now we state our main theorem. Actually we have the following:

Theorem 1. If $f$ satisfies (4), (7) (in the case of (7b) we prove this theorem only for $\left.\phi(\sigma)=b(1+\sigma)^{-1-b}\right)$ and $u_{0}^{*}(x)>0$ then $u^{*}=u\left(x, t ; \lambda^{*}\right)>0$ is a unique global-in-time solution to (1) and $u^{*}(x, t) \rightarrow \infty$ as $t \rightarrow \infty$ for all $x \in(-1,1), u_{x}^{*}( \pm 1, t) \rightarrow \mp \infty$ as $t \rightarrow \infty$, i.e. $u^{*}$ grows up globally.

The proof is given in the following lemmas and propositions:

Proposition 2. (Comparison). Let $u, v \in C^{2}\left(\Omega_{T}\right) \cap C\left(\bar{\Omega}_{T}\right)$, and $f$ satisfies (3) with

$$
\begin{aligned}
& u_{t}-\left(u^{n}\right)_{x x}-\frac{\lambda f(u)}{\left(\int_{-1}^{1} f(u) \mathrm{d} x\right)^{2}}<v_{t}-\left(v^{n}\right)_{x x}-\frac{\lambda f(v)}{\left(\int_{-1}^{1} f(v) \mathrm{d} x\right)^{2}}, \text { in } \Omega_{T} \\
& 0 \leq u( \pm 1, t)<v( \pm 1, t), \quad t>0 \\
& 0<u(x, 0)<v(x, 0), \quad x \in(-1,1)
\end{aligned}
$$

then $u(x, t)<v(x, t)$ for $(x, t) \in[-1,1] \times[0, T]$. In addition if $f$ is locally Lipschitz continuous and again $u(x, 0)>0$ then the proposition is true by substituting all $\leq$ for $<$.

Proof. We prove the proposition in two steps. Actually we give the proof for $\Omega \in \mathbb{R}^{N}, N \geq 1$ and for the Filtration equation i.e. instead of $u^{n}$ we work with $K(u)$.

First step: Let that $u, v$ satisfy (8), we shall show that $u(x, t)<v(x, t)$ for all $(x, t) \in \bar{\Omega} \times[0, T]$, for some $T>0$. Let $d=d(x, t)=v(x, t)-u(x, t)$ (see also [1]). We assume that the conclusion is false; that is, there exists a first $\bar{t} \in(0, T]$ such that $d(\bar{x}, \bar{t})=0$ for some $\bar{x} \in \Omega_{0} \subseteq \Omega$ and $d(x, \bar{t})>0$ for some $x \in \Omega_{+} \subseteq \Omega, \quad \Omega_{0} \cup \Omega_{+}=\bar{\Omega}$. Also, we have that $d(x, t)>0$ for $(x, t) \in \bar{\Omega} \times[0, \bar{t})$ and $d_{t}(\bar{x}, \bar{t}) \leq 0$, moreover, $d(\bar{x}, \bar{t})$ attains its minimum at $x=\bar{x}$, so $\nabla d(\bar{x}, \bar{t})=\nabla v(\bar{x}, \bar{t})-\nabla u(\bar{x}, \bar{t})=0$ and $\Delta d(\bar{x}, \bar{t}) \geq 0$. 
Thus, due to $(8 \mathrm{a})$, we have at $(\bar{x}, \bar{t})$ :

$$
\begin{aligned}
0 \geq & d_{t}(\bar{x}, \bar{t})=v_{t}(\bar{x}, \bar{t})-u_{t}(\bar{x}, \bar{t}) \\
> & K^{\prime}(v) \Delta v+K^{\prime \prime}(v)|\nabla v|^{2}-K^{\prime}(u) \Delta u-K^{\prime \prime}(u)|\nabla u|^{2}+N L T s \\
= & K^{\prime}(u) \Delta d(\bar{x}, \bar{t})+K^{\prime \prime}(u)\left(|\nabla v|^{2}-|\nabla u|^{2}\right)+\lambda\left(\frac{f(v(\bar{x}, \bar{t}))}{\left(\int_{\Omega} f(v(x, \bar{t})) \mathrm{d} x\right)^{2}}\right. \\
& \left.\quad-\frac{f(u(\bar{x}, \bar{t}))}{\left(\int_{\Omega} f(u(x, \bar{t})) \mathrm{d} x\right)^{2}}\right) \geq f(u(\bar{x}, \bar{t})) \frac{\left(\int_{\Omega} f(u(x, \bar{t})) \mathrm{d} x\right)^{2}-\left(\int_{\Omega} f(v(x, \bar{t})) \mathrm{d} x\right)^{2}}{\left(\left(\int_{\Omega} f(v(x, \bar{t})) \mathrm{d} x\right)^{2} \int_{\Omega} f(u(x, \bar{t})) \mathrm{d} x\right)^{2}} \\
= & f(u(\bar{x}, \bar{t})) \frac{\left[\int_{\Omega}(f(u(x, \bar{t}))+f(v(x, \bar{t}))) \mathrm{d} x\right]\left[\int_{\Omega}(f(u(x, \bar{t}))-f(v(x, \bar{t}))) \mathrm{d} x\right]}{\left[\int_{\Omega} f(v(x, \bar{t})) \mathrm{d} x \int_{\Omega} f(u(x, \bar{t})) \mathrm{d} x\right]^{2}} \geq 0,
\end{aligned}
$$

where NLTs is the difference of the non-local terms. The term with Laplacian is non-negative, the term with the gradient is zero and the difference of the non-local terms is non-negative due to the monotonicity of $f,\left(f^{\prime}<0\right)$. Hence, $0 \geq d_{t}(\bar{x}, \bar{t})>0$, which is a contradiction.

Second step: Let us now assume that $u, v$ satisfy $(8)$ but substituting $<$ for $\leq$. We shall show that $u(x, t) \leq v(x, t)$ for all $(x, t) \in \bar{\Omega} \times[0, T]$, for some $T>0$. Since $f \in C^{1}$, it is Lipschitz continuous and also one side Lipschitz continuous i.e. $f(a+b)-f(b) \leq L a$ where $L$ is a positive constant and $0<a<R$ for some $R$. Let $v^{\varepsilon}=v+\varepsilon e^{\sigma t}>v$ for some $\varepsilon>0$, actually we get $0<\varepsilon \ll 1$, $\sigma \gg 1$ and $\varepsilon e^{\sigma t}<\varepsilon e^{\sigma T}=R$ and $L$ fixed, then we have:

$$
\begin{aligned}
S\left(v^{\varepsilon}\right) \equiv & v_{t}^{\varepsilon}-\Delta K\left(v^{\varepsilon}\right)-\lambda \frac{f\left(v^{\varepsilon}\right)}{\left(\int_{\Omega} f\left(v^{\varepsilon}\right) \mathrm{d} x\right)^{2}} \\
= & \left.v_{t}+\varepsilon \sigma e^{\sigma t}-K^{\prime \prime}\left(v^{\varepsilon}\right)|\nabla v|^{2}-K^{\prime}\left(v^{\varepsilon}\right)\right) \Delta v-\lambda \frac{f\left(v^{\varepsilon}\right)}{\left(\int_{\Omega} f\left(v^{\varepsilon}\right) \mathrm{d} x\right)^{2}} \\
\geq & v_{t}-\Delta K(v)-\lambda \frac{f\left(v^{\varepsilon}\right)}{\left(\int_{\Omega} f\left(v^{\varepsilon}\right) \mathrm{d} x\right)^{2}}+\lambda \frac{f\left(v^{\varepsilon}\right)}{\left(\int_{\Omega} f(v) \mathrm{d} x\right)^{2}}-\lambda \frac{f(v)+L \varepsilon e^{\sigma t}}{\left(\int_{\Omega} f(v) \mathrm{d} x\right)^{2}} \\
& +\varepsilon \sigma e^{\sigma t}+\left(K^{\prime \prime}(v)-K^{\prime \prime}\left(v^{\varepsilon}\right)\right)|\nabla(v)|^{2}+\left(K^{\prime}(v)-K^{\prime}\left(v^{\varepsilon}\right)\right) \Delta v \\
= & S(v)+\varepsilon e^{\sigma t}\left[\sigma-\lambda f\left(v^{\varepsilon}\right) \frac{\left.\left[\int_{\Omega}\left(f\left(v^{\varepsilon}\right)+f(v)\right) \mathrm{d} x\right)\right]\left[\int_{\Omega} f^{\prime}(s) L \varepsilon e^{\sigma t} \mathrm{~d} x\right]}{\left[\int_{\Omega}\left(f\left(v^{\varepsilon}\right) \mathrm{d} x\right]^{2}\left[\int_{\Omega} f(v)\right) \mathrm{d} x\right]^{2}}\right. \\
& \left.-\lambda \frac{L}{\left(\int_{\Omega} f(v) \mathrm{d} x\right)^{2}}-K^{\prime \prime \prime}(v)|\nabla v|^{2}-K^{\prime \prime}(v) \Delta v\right]+O\left(\varepsilon^{2}\right) \\
> & S(v) \geq S(u),
\end{aligned}
$$

on the above we have used $f\left(v^{\varepsilon}\right) \leq f(v)+L \varepsilon e^{\sigma t}$. The last inequality is due to the fact that, $\sigma$ can be taken sufficient large $(\sigma \gg 1)$ while $R$ and $L$ are fixed and the function $v$ is bounded in $C^{2,1}\left(\bar{\Omega}_{T}\right)$. Then from first step we derive $v^{\varepsilon}>u$ and $v^{\varepsilon}>v$ for $\varepsilon \ll 1$. Now we have $v^{\varepsilon}=v+\varepsilon e^{\sigma t}>u$ for any $0<\varepsilon \ll 1$ and taking $\varepsilon \rightarrow 0$ we get $v \geq u$. This completes the proof.

A similar to Proposition 2 holds for weak upper (lower) solution to problem (1). A weak upper (lower) solution to problem (1) is any function $\bar{u}(\underline{u})$ which satisfies $(2)$ by substituting $\geq(\leq)$ for $=$. 
Proposition 3. (Comparison weak-form) Let $u, v \in C\left([0, T] ; L^{1}(\Omega) \cap L^{\infty}\left(\Omega_{T}\right)\right)$, and $f$ satisfies (3a), with $P(u ; \eta)<P(v ; \eta)$, then $u(x, t)<v(x, t)$ a.e. in $\Omega_{T}$. If in addition $f$ is locally Lipschitz continuous and again $u(x, 0)>0$ then the proposition is true by substituting all $\leq$ for $<$.

Lemma 4. (Local Existence). If $f$ satisfies (4a) and $u_{0}(x)>0$ then there exists a unique weak solution $u(x, t ; \lambda)$ to problem (1) to each $\lambda>0$ in $\Omega_{T}$ for some $T>0$. Moreover $u \in L^{\infty}\left(\Omega_{T}\right)$ and hence is classical.

Proof. Due to the fact that $f,-f^{\prime}>0$, a maximum principle holds for problem (1), this implies (Proposition 2) that if $u_{0}(x)>0$ then $u(x, t)>0$. Moreover, using comparison methods we can construct an increasing (decreasing) sequence of lower solutions $\underline{u}_{n}\left(\bar{u}_{n}\right)$, via an iteration scheme, such that $\underline{u}_{1}<$ $\ldots<\underline{u}_{n}<\cdots \leqslant \underline{u} \leqslant \bar{u} \leqslant \cdots<\bar{u}_{n}<\cdots<\bar{u}_{1}$ where $\underline{u}_{n} \nearrow \underline{u}$ and $\bar{u}_{n} \searrow \bar{u}$ as $n \rightarrow \infty$. The iteration scheme is defined by substituting $u_{n}$ for $u$ at the left hand-side and $u_{n-1}$ for $u$ at the right hand-side of the Eq. (1a), for more details see [10]. In addition, since $f$ is Lipschitz continuous we can prove (again by using maximum principle) that $\underline{u} \geqslant \bar{u}$ and hence $\underline{u}=u=\bar{u}$ where $u$ is the local in time unique solution to (1). This guarantees the local existence and uniqueness of $u$.

Remark 5. (i) The above lemma can be obtained by regularization of the solution $u_{\delta}$ which satisfies problem (1) where the term $\left(u_{\delta}^{n}\right)_{x x}=\left(u^{n}+\right.$ $\delta u)_{x x}$ is substituted for $\left(u^{n}\right)_{x x}$. The $u_{\delta}$-problem does not create degeneracy.

(ii) The degeneracy of problem (1) takes place only on the boundary $x= \pm 1$ and the solution is classical in $\Omega$, provided that $u$ is bounded.

(iii) Also the proof of Lemma 4 can be obtained by using the $L^{1}$-solution. It is well known that an $L^{1}$-solution which belongs to $L^{\infty}\left(\Omega_{T}\right)$ is also classical.

Lemma 6. The following statements hold:

(a) If $u_{0}(x)$ is a symmetric (even) function then the solution $u(x, t)$ to problem (1) is symmetric too.

(b) If $u_{0}$ is a strict lower solution to (3) then $u_{t}=u_{t}\left(x, t ; \lambda^{*}, u_{0}\right)>0$.

(c) If $u_{0} \geq u_{0}^{*}>0$ then $U=u\left(x, t ; \lambda^{*}, u_{0}\right) \geq u\left(x, t ; \lambda^{*}, u_{0}^{*}\right)=u^{*}>0$ where $U, u^{*}$ are solutions to (1).

Proof. (a) This is an immediate consequence of the symmetry of the problem.

(b) On using standard comparison arguments we get by Proposition 2 that $u(x, t) \geq u_{0}(x)$. Defining now $u^{\delta}(x, t)=u(x, t+\delta)$, then $u^{\delta}$ is a solution to problem (1) and so is an upper solution with $u^{\delta}(x, 0)=u(x, t) \geq u_{0}(x)$. Hence $u^{\delta}(x, t) \geq u(x, t)$ see also [1].

(c) This is a direct consequence of Proposition 2.

Lemma 7. Let that all conditions in Theorem 1 hold, then $u^{*}(x, t) \rightarrow \infty$ for all $x \in(-1,1)$ and $u_{x}( \pm 1, t) \rightarrow \mp \infty$ as $t \rightarrow t^{*}-\leq \infty$, for some $t^{*}>0$, where $t^{*}$ is the maximal existence time of $u^{*}$. 
Proof. It is enough to construct a lower solution $\underline{v}(x, t)$ to $u^{*}$-problem (problem (1) with $u(x, 0)=u_{0}^{*}(x)$ and $\left.\lambda=\lambda^{*}\right)$ such that $\underline{v}(x, t) \rightarrow \infty$ for all $x \in(-1,1)$ as $t \rightarrow T^{*}-\leq \infty$ for some $T^{*} \geq t^{*}>0$, where $T^{*}$ is the maximal existence time of $\underline{v}$. Therefore we get, $\underline{v}(x, t)=\underline{w}(x ; \mu(t))$ where $\underline{w}$ satisfies $(3)$, see also $[8,9,15]$. On using maximum principle we get $w_{\mu}>0$ (differentiating (3a) with respect to $\mu$ and setting $z=w^{n}$ in (3), then $z_{\mu}=n w^{n-1} w_{\mu}$ and $z_{\mu}^{\prime \prime}+\mu z_{\mu} f^{\prime}(w) /\left(n w^{n-1}\right)<0$ with $z_{\mu}( \pm 1, t)=0$ which implies $\left.z_{\mu} \geq 0\right)$. Now

$$
\begin{aligned}
\underline{v}_{t} & -\left(\underline{v}^{n}\right)_{x x}-\lambda^{*} \frac{f(\underline{v})}{\left(\int_{-1}^{1} f(\underline{v}) \mathrm{d} x\right)^{2}} \\
& =\underline{w}_{\mu}\left\{\dot{\mu}-\frac{f(\underline{w})}{\underline{w}_{\mu}\left(\int_{-1}^{1} f(\underline{w}) \mathrm{d} x\right)^{2}}\left[\lambda^{*}-\mu\left(\int_{-1}^{1} f(\underline{w}) \mathrm{d} x\right)^{2}\right]\right\} \\
& \leq \underline{w}_{\mu}\left\{\dot{\mu}-\left[\lambda^{*}-\mu\left(\int_{-1}^{1} f(\underline{w}) \mathrm{d} x\right)^{2}\right]\right\} \frac{1}{\left.\left(\int_{-1}^{1} f(\underline{w}) \mathrm{d} x\right)^{2}\right)} \inf _{(-1,1)} \frac{f\left(\underline{w}_{\mu}\right.}{\underline{w}_{\mu}} \leq 0
\end{aligned}
$$

where $\mu=\mu(t)$ satisfy:

$$
0<\dot{\mu} \leq \lambda^{*}-\mu\left(\int_{-1}^{1} f(\underline{w}) \mathrm{d} x\right)^{2} .
$$

On taking $\underline{w}(x ; \mu(0)) \leq u_{0}^{*}(x), \underline{v}$ is a lower solution to $u^{*}$-problem with $\underline{v}_{t}=$ $\underline{w}_{\mu} \dot{\mu}>0$, implying $\underline{v}(x, t)=\underline{w}(x ; \mu(t)) \rightarrow \infty$ for all $x \in(-1,1), \mu(t) \rightarrow \infty$ (or $\left.\bar{\lambda}(t) \rightarrow \lambda^{*}-\right)$ as $t \rightarrow T^{*} \leq \infty$, see also [9, p.204] and the response $\mu, \lambda$-diagram in $\left[15\right.$, p.448]. Hence $u(x, t) \rightarrow \infty$ as $t \rightarrow t^{*} \leq \infty$ uniformly in compact subsets of $(-1,1)$ and also $u_{x}( \pm 1, t) \rightarrow \mp \infty$ as $t \rightarrow t^{*} \leq \infty$. This completes the proof of the proposition.

Now, due to Lemma 6 , for any $u_{0}^{*}(x)$ it is enough for Theorem 1 to hold, to choose $u_{0}>u_{0}^{*}$ and prove that $U(x, t)=u\left(x, t ; \lambda^{*}, u_{0}\right)$ is a global in time solution of $u^{*}$. In addition we assume $u_{0}$ to be a positive, symmetric and a lower solution to (3) (for instance such a $u_{0}$ is: $u_{0}(x)=k>0$, for $|x| \leq \delta, k$ is a constant and $u_{0}(x)=w(y(x))$ with $y(x)=(|x|-\delta) /(1-\delta)$ for $0<\delta \leq|x| \leq 1$ where $w(y(x))$ satisfies problem $(3))$.

On taking now $U$ to be a solution to (1) and make the "pressure transformation": $0<z=z(x, t)=U^{n}(x, t)$, then $n U_{t}=z_{t} z^{-q}$ where $0<q=1-1 / n$. Thus problem (1) becomes the "pressure problem":

$$
\begin{aligned}
& z_{t}=n z^{q}\left(z_{x x}+\lambda^{*} \frac{\phi(z)}{\left(\int_{-1}^{1} \phi(z) \mathrm{d} x\right)^{2}}\right), \quad x \in(-1,1), \quad t>0, \\
& z(1, t)=z(-1, t)=0, \quad t>0, \\
& z(x, 0)=z_{0}(x)>0, \quad x \in(-1,1),
\end{aligned}
$$

where the function $\phi$ is defined: $\phi(z)=f\left(z^{1 / n}\right)=f(U)>0$ with $\phi^{\prime}(z)<0$, for $z \geq 0$. The solution $z(x, t)$ of $(9)$ satisfies: $z(x, t)$ is symmetric with respect 
to $x, \max _{x} z(x, t)=z(0, t)=M(t)=U^{n}(0, t)$ and $z_{t}>0$ since $U_{t}>0$, due to Lemma 6 .

Moreover due to $(4 \mathrm{~b})$ we get:

$$
\int_{0}^{\infty} \phi(z) \mathrm{d} z=\int_{0}^{\infty} f\left(z^{1 / n}\right) \mathrm{d} z=n \int_{0}^{\infty} U^{n-1} f(U) \mathrm{d} U<\infty .
$$

Finally, the "pressure equation" gives:

$$
z_{t} \leq n M^{q}\left(z_{x x}+\lambda^{*} \frac{\phi(z)}{\left(\int_{-1}^{1} \phi(z) \mathrm{d} x\right)^{2}}\right) .
$$

Now we introduce the $\bar{u}$-problem:

$$
\begin{aligned}
& \bar{u}_{t}=n M^{q}\left(\bar{u}_{x x}+\lambda^{*} \frac{\phi(\bar{u})}{\left(\int_{-1}^{1} \phi(\bar{u}) \mathrm{d} x\right)^{2}}\right), \quad x \in(-1,1), \quad t>0, \\
& \bar{u}(1, t)=\bar{u}(-1, t)=0, \quad t>0, \\
& \bar{u}(x, 0)=\bar{u}_{0}(x)>z_{0}(x)=u_{0}^{n}(x)>0, \quad x \in(-1,1),
\end{aligned}
$$

where $\bar{u}_{0}$ is taken to be symmetric and a lower solution to (12a), (12b); hence $\bar{u}_{t}>0$. Then $z$ is a lower solution to $\bar{u}$-problem with $z(x, t)<\bar{u}(x, t)$ and thus $M(t)<\bar{M}(t)=\max _{x} \bar{u}(x, t)=\bar{u}(0, t)$.

We proceed by rescaling the time so that:

$$
\frac{d \tau}{d t}=n M^{q}(t)
$$

and $\bar{u}(x, t)=\widehat{u}(x, \tau)$ with $\bar{u}(0, t)=\bar{M}(t)=\widehat{u}(0, \tau)=\widehat{M}(\tau)$. Thus we have concluded to the following $\widehat{u}$-problem:

$$
\begin{aligned}
& \widehat{u}_{\tau}=\widehat{u}_{x x}+\lambda^{*} \frac{\phi(\widehat{u})}{\left(\int_{-1}^{1} \phi(\widehat{u}) \mathrm{d} x\right)^{2}}, \quad x \in(-1,1), \quad \tau>0, \\
& \widehat{u}(1, \tau)=\widehat{u}(-1, \tau)=0, \quad \tau>0, \\
& \widehat{u}(x, 0)=\widehat{u}_{0}(x), \quad x \in(-1,1) .
\end{aligned}
$$

Proposition 8. If $\phi(s)=f\left(s^{1 / n}\right)$ satisfies (4), (7) (in the case of (7b) we have this proposition only for $\left.\phi(\sigma)=b(1+\sigma)^{-1-b}\right)$ and $\widehat{u}_{0}(x)>0$ then then $\widehat{u}(x, \tau)>0$ is a unique global-in-time solution to (14) and $\widehat{u}(x, \tau) \rightarrow \infty$ as $\tau \rightarrow \infty$ for all $x \in(-1,1), \widehat{u}_{x}( \pm 1, \tau) \rightarrow \mp \infty$ as $\tau \rightarrow \infty$ i.e. $\widehat{u}$ diverges globally in $x$ or equivalently $\widehat{u}$ grows up globally.

Proof. For the proof see [6, p. 795, Prop. 2],[11].

Remark 9. The method used to prove Proposition 8 in [6], is based on the construction of an upper solution to $u^{*}$ which is global in time. It seems that this method works only for the $\Delta u$ and not for $\Delta u^{n}$. Any such construction, for the case $\Delta u^{n}$, leads to an upper solution which blows-up in finite time and then it is useless for our purpose. This is the reason why, in this work, we introduce a different method. 
Hence relation (13) and Proposition 8 imply:

$$
\widehat{u}(x, \tau)=\bar{u}(x, t) \rightarrow \infty,
$$

for each $x \in(-1,1)$ as $\tau \rightarrow \infty$ and as $t \rightarrow t^{*} \leq \infty$.

In order to prove Theorem 1 , it is required to show that $t^{*}=\infty$. Therefore we assume that $t^{*}<\infty$ and get a contradiction. For this purpose we need the following lemma:

Lemma 10. (Flat Blow-up). Let $\bar{u}(x, t)$ be the solution to problem (12) which blows up in finite time $t^{*}$, then the blow-up is flat on $[-\delta, \delta], 0<\delta<1$, i.e. $\bar{u}(x, t) \sim \bar{M}(t)$, for $|x| \leq \delta, 0<1-\delta \ll 1$ and $0<t^{*}-t \ll 1$.

Proof. For the proof we use similar ideas as in [9, p. 215]. We introduce again an auxiliary problem:

$$
\begin{gathered}
H(\widetilde{u}) \equiv \widetilde{u}_{t}-\widetilde{u}_{x x}-\bar{g}(t) \phi(\bar{M})=0, \quad x \in(-1,1), \quad t>t_{0} \geq 0, \\
\widetilde{u}(1, t)=\widetilde{u}(-1, t)=0, \quad t>t_{0}, \\
\widetilde{u}\left(x, t_{0}\right)=\widetilde{u}_{0}(x)=0, \quad x \in(-1,1),
\end{gathered}
$$

where $\bar{g}(t)=\lambda^{*} /\left(\int_{-1}^{1} \phi(\bar{u}) \mathrm{d} x\right)^{2}$ and for some $t_{0} \geq 0$.

From (12), since $\phi$ is decreasing, we get:

$$
0<\bar{M}^{\prime}(t) \leq h(t) \phi(\bar{M}(t)) \leq h(t) \phi\left(\bar{M}\left(t_{0}\right)\right), \quad t \geq t_{0} \geq 0,
$$

where $h(t)=n M^{q}(t) \bar{g}(t), \bar{M}(t)=\max _{x} \bar{u}(x, t)=\bar{u}(0, t)$, since $\bar{u}_{t}>0$ and $\bar{u}_{x x} \leq$ 0 at any point that $\bar{u}$ takes the maximum value $\bar{M}$. Since $\bar{u}$ blows up, then $\bar{M}(t) \rightarrow \infty$ as $t \rightarrow t^{*}-\left(\right.$ if $_{\liminf _{t \rightarrow t^{*}-}} \bar{M}(t)<\infty$, then $\liminf _{t \rightarrow t^{*}-} M(t)<$ $\infty$, which implies that there exists $t_{n}$ such that the lower solution $\underline{v}\left(0, \mu\left(t_{n}\right)\right)=$ $w\left(0, \mu\left(t_{n}\right)\right)<\infty$ contradicting to Lemma 7$)$, this also implies that $h(t)=$ $n M^{q}(t) \bar{g}(t), \bar{g}(t) \rightarrow \infty$ as $t \rightarrow t^{*}-$, (otherwise $\bar{u}$ does not blow-up, since $\phi$ is decreasing and necessarily bounded), since the blow-up of $\bar{u}$ is global i.e. $\bar{u}(x, t) \rightarrow \infty$ as $t \rightarrow \infty$ for all $x \in(-1,1)$. Moreover, integrating (17) we get $\int^{t} h(s) \phi(\bar{M}(s)) \mathrm{d} s \rightarrow \infty$ and $\int^{t} h(s) \mathrm{d} s \rightarrow \infty$ as $t \rightarrow t^{*}-$. Similarly we have: $\int^{t} h(s) \phi(\bar{M}(s)) \mathrm{d} s=\int^{t} n M^{q}(s) \bar{g}(s) \phi(\bar{M}(s)) \mathrm{d} s \leq \int^{t} n \bar{M}^{q}(s) \bar{g}(s) \phi(\bar{M}(s)) \mathrm{d} s \leq$ $K \int^{t} \bar{g}(s) \mathrm{d} s$, since $q<1, M(t) \leq \bar{M}(t), \bar{M} \phi(\bar{M}) \rightarrow 0$ as $\bar{M} \rightarrow \infty$ and $\bar{M} \phi(\bar{M})<K$ for $\bar{M} \gg 1$, hence $\int^{t} \bar{g}(s) \mathrm{d} s \rightarrow \infty \quad$ as $t \rightarrow t^{*}-$.

We also introduce the initial value problem:

$$
V^{\prime}(t)=h(t) \phi(\bar{M}(t)), \quad V\left(t_{0}\right)=0,
$$

this implies $V(t)=\int_{t_{0}}^{t} h(s) \phi(\bar{M}(s)) \mathrm{d} s \rightarrow \infty$ as $t \rightarrow t^{*}-$ and $t_{0}$ is defined (Lemma 7) so that:

$$
1 \leq n \widetilde{M}^{q}\left(t_{0}\right) \leq n \bar{M}^{q}\left(t_{0}\right) \leq n \bar{M}^{q}(t),
$$

since $\bar{M}(t) \geq M(t), \widetilde{M}(t)=\max _{x} \widetilde{u}(x, t)$ (see below that $\bar{u} \geq \widetilde{u}$ ) and $M^{\prime}(t)>$ $0, z_{t}>0$ for $t_{0}<t<t^{*}$. 
Relations (17), (18) also imply:

$$
\bar{M}(t)-\bar{M}\left(t_{0}\right) \leq V(t), \quad t \geq t_{0}, \quad \text { or } \quad \bar{M}(t) \lesssim V(t),
$$

for $\bar{M} \gg 1$ or for $0<t^{*}-t \ll 1$.

In addition the solution to (12) is an upper solution to (16). Indeed, since $\bar{u}_{t}>0$ then $\left(\bar{u}_{x x}+\bar{g}(t) \phi(\bar{u})\right)>0$; hence from (19) and for $x \in(-1,1), t \geq t_{0}$ we have:

$$
\begin{aligned}
H(\bar{u})=\bar{u}_{t}-\bar{u}_{x x}-\bar{g}(t) \phi(\bar{M}) & \geq \bar{u}_{t}-\left(\bar{u}_{x x}+\bar{g}(t) \phi(\bar{u})\right) \\
& \geq \bar{u}_{t}-n M^{q}\left(\bar{u}_{x x}+\bar{g}(t) \phi(\bar{u})\right)=0=H(\widetilde{u}) .
\end{aligned}
$$

Also $\widetilde{u}( \pm 1, t)=\bar{u}( \pm 1, t)=0$ and $\widetilde{u}\left(x, t_{0}\right)=0<\bar{u}\left(x, t_{0}\right)$, due to problem (12) and that $u_{0}(x)>0$.

Hence $\bar{u}(x, t) \geq \widetilde{u}(x, t)$ and therefore we have:

$$
\bar{M}(t) \geq \bar{u}(x, t) \geq \widetilde{u}(x, t), \quad(x, t) \in(-1,1) \times\left(t_{0}, t^{*}\right) .
$$

On the other hand, for $t>t_{0}, \widetilde{u}$ satisfies:

$$
\widetilde{u}(x, t)=V(t)+\int_{t_{0}}^{t} V(s)\left[G_{y}(x, 1, t-s)-G_{y}(x,-1, t-s)\right] \mathrm{d} s,
$$

where $G(x, y, t-s)$ is the Green's function for the heat equation in $(-1,1)$ with Dirichlet boundary conditions. Also we note that $\bar{u}, \widetilde{u} \rightarrow \infty$ as $t \rightarrow t^{*}-$.

Now, for $x \in \bar{I}_{\delta} \equiv[-1+\delta, 1-\delta]$ and any fixed $0<\delta<1$, the following asymptotic estimate holds:

$$
\widetilde{u}(x, t) \sim V(t), \quad 0<t^{*}-t \ll 1, \quad t>t_{0},
$$

since for any fixed $x \in \bar{I}_{\delta}$ the second term on the right hand side of (22) is much smaller than the first one, the latter is due to the form of the Green's function, see [9]. This also hold, on taking $\delta=\delta(t) \rightarrow 0$, as long as it goes to zero slowly enough. Finally $(20),(21)$, (23), for $x \in \bar{I}_{\delta}, 0<t^{*}-t \ll 1, t>t_{0}$, give:

$$
\bar{M}(t) \geq \bar{u}(x, t) \geq \widetilde{u}(x, t) \sim V(t) \gtrsim \bar{M}(t)
$$

and the proof of the lemma is now completed.

Now using (15), Proposition 8 and Lemma 10 we obtain, $\bar{u}(x, t) \sim \bar{M}(t)=\widehat{M}(\tau) \rightarrow \infty$ for $0<t^{*}-t \ll 1$ or $0<1 / \tau \ll 1, \forall x \in(-1,1)$. Hence, for the $\widehat{u}$-problem we get a flat grow-up, which implies:

$$
\widehat{M}^{\prime}(\tau)=\frac{d \widehat{M}(\tau)}{d \tau}=\lambda^{*} \frac{\phi(\widehat{M})}{\widehat{I}^{2}(\tau)}=g(\tau ; \widehat{u}) \phi(\widehat{M}) \sim \lambda^{*} \frac{\phi(\widehat{M})}{4 \phi^{2}(\widehat{M})}=\frac{\lambda^{*}}{4 \phi(\widehat{M}(\tau))},
$$

for $0<t^{*}-t \ll 1$ or $0<1 / \tau \ll 1$. Thus $\phi(\widehat{M}) d \widehat{M} \sim \lambda^{*} d \tau / 4, \quad \tau \gg 1$, provided that the dominant contribution to the integral does not come from the boundary layers. 
Integrating now we derive:

$$
\frac{\lambda^{*}}{4} \tau \sim \int^{\widehat{M}(\tau)} \phi(s) \mathrm{d} s<\int_{0}^{\infty} \phi(s) \mathrm{d} s<\infty .
$$

The last relation is a contradiction since $\tau$ is unbounded and hence $t^{*}=\infty$.

Now it remains to calculate the integral in the case that a significant (but dominant) contribution comes from the boundary layers. To find these two contributions from the boundary layers to the integral $I$, we follow similar analysis as in the case $\lambda>\lambda^{*}$, see $[9$, p. 216].

For simplicity, we only consider the boundary layer at $x=1$. If the boundary layer has width $O(\delta)$, for some $\delta \ll 1$, to compare in pairs the terms of the equation we rescale again as follow, actually we have:

$$
\begin{aligned}
y & =\frac{x}{\delta}, \quad \frac{d T}{d \tau}=\frac{1}{t^{*}-t}, \quad \frac{d T}{d t}=\frac{n M^{q}(t)}{t^{*}-t} \quad \text { and } \\
\widehat{u}(x, \tau) & =\widetilde{U}(y, T), \quad \widetilde{M}(\tau)=\widetilde{M}(T)=\sup _{y} \widetilde{U}(y, T) .
\end{aligned}
$$

Then Eq. (1) becomes

$$
\widetilde{U}_{T} \frac{1}{t^{*}-t}=\frac{1}{\delta^{2}} \widetilde{U}_{y y}+g(T ; \widetilde{U}) \phi(\widetilde{U}) .
$$

Again following the same analysis as in [9] we have:

(i) If $g(T ; \widetilde{U})=g(t ; \bar{u}) \ll\left(t^{*}-t\right)^{-1}$, then $O(\delta)=O\left(\left(t^{*}-t\right)^{1 / 2}\right) \ll 1$ and

$$
I=\widetilde{I}(T)=O\left((g(T ; \widetilde{U}))^{-1 / 2}\right) \gg\left(t^{*}-t\right)^{1 / 2} .
$$

This suggests that the contribution to the integral $I$ from the boundary layer, is $O(\delta)=O\left(\left(t^{*}-t\right)^{1 / 2}\right) \ll 1$ and therefore finally the main core dominates as in the previous case. Namely $I \sim 2 \phi(\widehat{M})$; hence again we get a contradiction $(\tau<\infty)$, and hence $t^{*}=\infty$.

(ii) If $g(T ; \widetilde{U})=g(t ; \bar{u})=g(\tau ; \widehat{u})=\widehat{g}(\tau) \sim O\left(\left(t^{*}-t\right)^{-1}\right)$, again we have

$$
2 \phi(\widehat{M})<\widehat{I}(\tau)=\sqrt{\lambda^{*} / \widehat{g}(\tau)},
$$

and wherever we have flat grow-up, (25) gives

$$
\begin{aligned}
& \widehat{M}^{\prime}(\tau) \sim g(\tau ; \widehat{u}) \phi(\widehat{M}) \leq \frac{1}{2}\left(\lambda^{*} \widehat{g}(\tau)\right)^{1 / 2}=O\left(\left(t^{*}-t\right)^{-1 / 2}\right), \\
& \frac{d \bar{M}}{d t} \frac{1}{n \bar{M}} \leq \frac{d \bar{M}}{d t} \frac{1}{n \bar{M}^{q}} \leq \frac{d \widehat{M}}{d \tau}=\frac{d \bar{M}}{d t} \frac{1}{n M^{q}} \leq O\left(\left(t^{*}-t\right)^{-1 / 2}\right),
\end{aligned}
$$

or (since $M(t) \leq \bar{M}(t)$ and $q<1$ ) we get

$$
\ln \frac{\bar{M}(t)}{\bar{M}\left(t_{0}\right)} \leq K\left[\left(t^{*}-t_{0}\right)^{1 / 2}-\left(t^{*}-t\right)^{1 / 2}\right] .
$$

On taking now $t \rightarrow t^{*}-<\infty$ we get again a contradiction and hence $t^{*}=\infty$. 
(iii) Now if $g(T ; \widetilde{U}) \gg\left(t^{*}-t\right)^{-1}$, also we have $g(T ; \widetilde{U})=g(\tau ; \widehat{u})=\delta^{-2} \gg$ $\left(t^{*}-t\right)^{-1}$. These suggest that $\widehat{u}_{\tau}$ is negligible and the key balance is

$$
-\widehat{u}_{x x} \sim \frac{\lambda^{*} \phi(\widehat{u})}{\left(\int_{-1}^{1} \phi(\widehat{u}) \mathrm{d} x\right)^{2}}=g(\tau ; \widehat{u}) \phi(\widehat{u}), \quad \text { for } \tau \rightarrow \infty, \widehat{u} \rightarrow \infty,
$$

(which might be thought of as coming from the approximate equation in the boundary layers which occur for $\lambda>\lambda^{*}$, and these boundary layers having expanded to fill the whole of $-1<x<1$ if $\lambda \rightarrow \lambda^{*}+$ ).

To leading order then,

$$
\widehat{u} \sim W(x ; \widehat{g}(\tau))
$$

where

$$
\frac{d^{2} W}{d x^{2}}+\widehat{g}(\tau) \phi(W)=0, \text { for }-1<x<1, W=0 \text { at } x= \pm 1 .
$$

As in the case of $\lambda>\lambda^{*},[9$, p. 217]), the contribution from the boundary layers is

$$
\sim \frac{2}{\sqrt{\widehat{g}(\tau)}} \int_{0}^{\infty} \phi(\widehat{u}(1-\sqrt{\sigma / \widehat{g}(\tau)}, \tau)) \mathrm{d} \sigma=\sqrt{\lambda^{*} / \widehat{g}(\tau)} .
$$

This gives

$$
\left(\frac{\lambda^{*}}{\widehat{g}(\tau)}\right)^{1 / 2} \sim 2 \phi(\widehat{M})+\left(\frac{\lambda^{*}}{\widehat{g}(\tau)}\right)^{1 / 2}
$$

which implies

$$
\phi(\widehat{M}) \sim 0 \text { and } \phi(\widehat{M})=o\left(\sqrt{\left.\lambda^{*} / \widehat{g}(\tau)\right)^{1 / 2}}\right) .
$$

The latter asymptotic information is not helpful in estimating the integral $I$, (as it is for the blow-up case when $\lambda>\lambda^{*}$, [9])

Therefore we use a different method.

(a) If the function $\phi$ satisfies (7a), we have $g(\tau ; \widehat{u}) \phi(\widehat{M})=\mu(\widehat{M}) \phi(\widehat{M}) \sim C_{0}$ for $\tau \gg 1$ or $\widehat{M} \gg 1$. Then from (25) we get:

$$
\frac{d \bar{M}}{d t} \frac{1}{n M^{q}(t)}=\frac{d \widehat{M}}{d \tau} \sim g(\tau ; \widehat{u}) \phi(\widehat{M}) \sim C_{0}
$$

or

$$
\frac{d \bar{M}}{d t} \lesssim C_{0} n \bar{M}^{q}(t) \leq k \bar{M}(t),
$$

$(M<\bar{M}, q=1-1 / n<1)$. On integrating we get:

$$
\ln \frac{\bar{M}(t)}{\bar{M}\left(t_{0}\right)} \lesssim k\left(t-t_{0}\right), \quad \bar{M}\left(t_{0}\right) \gg 1,
$$

and as $t \rightarrow t^{*}-, \bar{M}(t) \rightarrow \infty$ which again gives a contradiction. Hence $t^{*}=\infty$. 
(b) If the function $\phi$ satisfies (7b), we have $\mu(\sigma) \phi(\sigma) \sim C_{1} \sigma$ for $\sigma \gg 1$. Then, Eq. (28) is written, for $W(x ; \widehat{g}(\tau)) \gg 1$ (away from the boundaries):

$\frac{d^{2} W}{d x^{2}}+C_{1} W \sim 0$, for $-1+\delta<x<1-\delta, W=0$ at $x= \pm 1, \delta>0$.

This implies $W(x ; \widehat{g}(\tau))=a(\tau) \cos k x+b(\tau) \sin k x$ where $k^{2}=C_{1}, a(\tau)=$ $\widehat{M}(t)$ and $x \in(-1+\delta, 1-\delta)$, but the latter contradicts the flat grow-up of $\widehat{u} \sim W(x ; \widehat{g}(\tau))$ in the main core. This result holds provided that $(27)$ is true. As it is not trivial to verify the validity of (27) we give two special cases, see also [6].

- Exponential decay $\left(\phi(s)=e^{-s}\right)$.

For this case we have:

$$
\frac{d \widehat{M}}{d \tau}=\widehat{g}(\tau) \phi(\widehat{M}) \sim \frac{\pi^{2}}{2} e^{\widehat{M}} e^{-\widehat{M}}=\frac{\pi^{2}}{2},
$$

and integrating again we get a contradiction as in (iiia). Hence $t^{*}=\infty$.

- Power-law decay $\left(\phi(s)=b(1+s)^{-(1+b)}, b>0\right)$.

Again following the same analysis of $[6]$ we get:

$$
\widehat{u}(x, \tau) \sim \widehat{M}(\tau) W(x) \sim c_{2} \tau^{2 / b} w(x), \quad \text { as } \quad \tau \rightarrow \infty, \quad x \in(-1,1),
$$

contradicting the flat grow-up of $\widehat{u}(x, \tau)$, as in (iiib).

Finally in all above cases we get a contradiction. This fact implies that $\bar{u}$ and $u^{*}$ are both global-in-time solutions, with $u^{*}$ the solution of problem (12).

Proof. (of Theorem 1): By Lemma 4 we get a local-in-time unique solution $u^{*}$ to $(1)$. By Lemma $7, u^{*}(x, t)$ is unbounded, actually $u^{*}(x, t) \rightarrow \infty$ for all $x \in(-1,1)$ as $t \rightarrow t^{*}-\leq \infty$. Then by Lemma 7 , Proposition 8 and the above asymptotic analysis, we obtain that $u^{*}$ is global-in-time, actually we have,

$$
u^{*}(x, t) \leq \bar{u}(x, t)=\widehat{u}(x, \tau) \rightarrow \infty, \quad x \in(-1,1), \quad \text { as } \quad t, \tau \rightarrow \infty .
$$

Moreover $u_{x}^{*}( \pm 1, t) \rightarrow \mp \infty$ as $t \rightarrow \infty$. This completes the proof of the Theorem.

\section{Discussion}

In this problem we know, as in [15], that there exist a critical value $\lambda^{*}$ of the parameter $\lambda$, such that for $\lambda>\lambda^{*}, u=u(x, t ; \lambda)$, the solution to (1), blows-up globally in finite time and there is not any steady-state solution for $\lambda \geq \lambda^{*}$, while for $\lambda \in\left(0, \lambda^{*}\right)$ the solution $u(x, t ; \lambda)$ is a global-in-time bounded solution so that $u(x, t ; \lambda) \rightarrow w(x ; \lambda)$ as $t \rightarrow \infty$, where $w$ is the unique solution to (3).

In the present work we have shown that for $\lambda=\lambda^{*}$, the critical solution $u^{*}=u\left(x, t ; \lambda^{*}\right)$, under the assumption of Theorem 1 , "grows-up" globally, this means that $u^{*}$ is a global-in-time unbounded solution which diverges globally $\left(u(x, t) \rightarrow \infty\right.$ for all $x \in(-1,1), u_{x}( \pm 1, t) \rightarrow \mp \infty$ as $\left.t \rightarrow \infty\right)$.

As a future work, it will be interesting to find exactly how this solution $u^{*}$ diverges (asymptotic behaviour). A similar result has been examined in [6] 
and we conjecture that this method can also be applied to our solution $u^{*}$. An upcoming work it will be also to study the same problem in $N$-dimensions.

\section{Acknowledgements}

The authors would like to thank Professor A. A. Lacey for several fruitful discussions.

\section{References}

[1] Bebernes, J.W., Eberly, D.: Mathematical problems from combustion theory. Appl. Math. Sci. 83. Springer, Berlin (1989)

[2] Bebernes, J.W., Lacey, A.A.: Global existence and finite-time blow-up for a class of non-local parabolic problems. Adv. Differ. Equ. 2, 927-953 (1997)

[3] Bebernes, J.W., Talaga, P.: Non-local problems modelling shear banding. Commun. Appl. Nonlinear Anal. 3, 79-103 (1996)

[4] Bebernes, J.W., Li, C., Talaga, P.: Single-point blow-up for non-local parabolic problems. Physica D 134, 48-60 (1999)

[5] Caglioti, E., Lions, P.-L., Marchioro, C., Pulvirenti, M.: A special class of stationary flows for two-dimensional Euler equations: a statistical mechanics description. Commun. Math. Phys. 143, 501-525 (1992)

[6] Kavallaris, N.I., Lacey, A.A., Tzanetis, D.E.: Global existence and divergence of critical solutions of a non-local parabolic problem in Ohmic heating process. Nonlinear Anal. TMA 58, 787-812 (2004)

[7] Krzywicki, A., Nadzieja, T.: Some results concerning the Poisson-Boltzmann equation. Zastosowania Mat. (Appl. Math. (Warsaw)) 21, 265-272 (1991)

[8] Lacey, A.A.: Thermal runaway in a non-local problem modelling Ohmic heating. Part I: Model derivation and some specail cases. Eur. J. Appl. Math. 6, 127$144(1995)$

[9] Lacey, A.A.: Thermal runaway in a non-local problem modelling Ohmic heating. Part II: General proof of blow-up and asymptotics of runaway. Eur. J. Appl. Math. 6, 201-224 (1995)

[10] Latos, E.A., Tzanetis, D.E.: Existence and blow-up of solutions for a non-local filtration and porous medium problem. In: Proceedings of the Edinburgh Mathmatical Society (2009, in press)

[11] Liu, Q., Liang, F., Li, Y.: Asymptotic behavior of a nonlocal parabolic problem in Ohmic heating process. Eur. J. Appl. Math. 20(3), 247-267 (2009)

[12] Ockendon, J., Howison, S., Lacey, A., Movchan, A.: Applied Partial Differential Equations. Oxford Univercity Press, Oxford (1999) 
[13] Sattinger, D.H.: Monotone methods in nonlinear elliptic and parabolic boundary value problems. Indiana Univ. Math. J. 21, 979-1000 (1972)

[14] Tzanetis, D.E.: Blow-up of radially symmetric solutions of a non-local problem modelling Ohmic heating. Electron. J. Differ. Equ. 11, 1-26 (2002)

[15] Tzanetis, D.E., Vlamos, P.M.: A nonlocal problem modelling Ohmic heating with variable thermal conductivity. Nonlinear Anal. RWA 2, 443-454 (2001)

E. A. Latos, D. E. Tzanetis

Department of Mathematics, School of Applied Mathematical and Physical Sciences, National Technical University of Athens, Zografou Campus,

15780 Athens, Greece

e-mail: latev@mail.ntua.gr

D. E. Tzanetis

e-mail: dtzan@math.ntua.gr

Received: 08 October 2008.

Accepted: 12 October 2009. 